\title{
Targeting FR $\beta+$ tumor associated macrophages with car T cells in ovarian cancer
}

\author{
Rachel C Lynn ${ }^{1 *}$, Takami Matsuyama², Daniel J Powell ${ }^{1}$ \\ From 30th Annual Meeting and Associated Programs of the Society for Immunotherapy of Cancer (SITC 2015) \\ National Harbor, MD, USA. 4-8 November 2015
}

\begin{abstract}
Background
Chimeric antigen receptor (CAR) T cell therapy has shown dramatic clinical success in CD19+ leukemia and lymphoma patients. However, CAR T cell therapy for epithelial cancers has been less successful. Developing effective CAR T cell therapies for other types of cancer may involve overcoming several obstacles associated with solid tumors. Poor blood supply and dense stromal components can hinder access of CAR T cells to tumor cells. In addition, immunosuppressive elements in the tumor microenvironment may suppress $\mathrm{T}$ cell functional activity. Tumor associated macrophages (TAMs) have been identified as key pro-tumor players in the microenvironment. Tumors co-opt macrophage function to help promote tumor growth ("M2" polarization) via many diverse mechanisms including promoting angiogenesis, metastasis, and immune evasion. Indeed, the presence of TAMs correlates with worse overall prognosis in many types of cancer, including ovarian cancer patients. We hypothesized that utilizing the power of CAR T cells to target TAMs could be an effective way to improve CAR $\mathrm{T}$ cell therapy in epithelial cancer.
\end{abstract}

\section{Methods}

Folate receptor beta (FR $\beta$ ), a GPI-linked membrane protein that mediates folic acid uptake, has previously been described on TAMs in human cancer patients and mouse models of cancer. We found that FR $\beta$ is highly expressed in monocyte-derived M2 polarized macrophages. Using high affinity human FR $\beta$-specific CAR $T$ cells (m923), we observed significant IFN- $\gamma$ secretion and dose-dependent cytolysis of M2 polarized macrophages. When co-cultured with FR $\beta(-)$ human ovarian cancer cell line SKOV3, m923 CAR T cells mediated bystander killing of FR $\beta(-)$ tumor cells when FR $\beta+$ M2

${ }^{1}$ University of Pennsylvania, Philadelphia, PA, USA

Full list of author information is available at the end of the article polarized macrophages were present. Next, we confirmed high expression of FR $\beta$ in TAMs from primary ovarian cancer. m923 CAR T cells displayed specific activation in the presence of primary ovarian cancer, supporting the hypothesis that m923 CAR T cells could be utilized to eliminate FR $\beta$ + TAMs in patients.

To test our hypothesis in preclinical mouse models, we developed a mouse FR $\beta$-specific CAR (CL10).

\section{Results}

Using the implantable murine ovarian carcinoma ID8, we confirmed expression of FR $\beta$ in $\mathrm{F} 4 / 80^{+} / \mathrm{CD} 204^{+}$ TAMs isolated from peritoneal tumor ascites by flow cytometry. In addition, CL10 CAR T cells displayed significant specific reactivity against isolated $C D 11 b^{+}$ TAMs. Our preliminary data suggest that adoptive transfer of CL10 CAR T cells into ID8 tumor-bearing mice results in destruction of FR $\beta$ + TAMs and delayed tumor growth. Future experiments will determine whether combining CL10 with tumor-directed CAR $\mathrm{T}$ cells will result in synergistic anti-tumor effects.

\section{Conclusions}

In conclusion, our results suggest targeting TAMs with CAR T cells may be a novel way to enhance CAR therapy for solid tumors.

\section{Authors' details \\ ${ }^{1}$ University of Pennsylvania, Philadelphia, PA, USA. ${ }^{2}$ Department of Immunology, Graduate School of Medical and Dental Sciences, Kagoshima University, Kagoshima, Japan.}

Published: 4 November 2015

doi:10.1186/2051-1426-3-S2-P32

Cite this article as: Lynn et al:: Targeting FR $3+$ tumor associated

macrophages with car T cells in ovarian cancer. Journal for

ImmunoTherapy of Cancer 2015 3(Suppl 2):P32. 